\title{
Identification of genes with altered expression in male and female Schlager hypertensive mice
}

\author{
Christine L Chiu ${ }^{1 *}$, Kristy L Jacksonn ${ }^{2,3}$, Nerissa L Hearn ${ }^{1}$, Nicole Steiner ${ }^{1}$, Geoffrey A Head ${ }^{2,3}$ and Joanne M Lind ${ }^{1}$
}

\begin{abstract}
Background: Numerous studies have shown sex differences in the onset and severity of hypertension. Despite these sex-differences the majority of animal studies are carried out in males. This study investigated expression changes in both male and female hypertensive mouse kidneys to identify common mechanisms that may be involved in the development of hypertension.
\end{abstract}

Methods: The Schlager hypertensive mouse model (BPH/2J) and its normotensive control (BPN/3J) were used in this study. Radiotelemetry was performed on 12 to 13 week old BPH/2J and BPN/3J male and female animals. Affymetrix GeneChip Mouse Gene 1.0 ST Arrays were performed in kidney tissue from 12 week old BPH/2J and BPN/3J male and female mice ( $n=6 /$ group). Genes that were differentially expressed in both male and female datasets were validated using $\mathrm{qPCR}$.

Results: Systolic arterial pressure and heart rate was significantly higher in BPH/2J mice compared with BPN/3J mice in both males and females. Microarray analysis identified 153 differentially expressed genes that were common between males and females (70 upregulated and 83 downregulated). We validated 15 genes by qPCR. Genes involved in sympathetic activity (Hdc, Cndp2), vascular ageing (Edn3), and telomere maintenance (Mcm6) were identified as being differentially expressed between BPH/2J and BPN/3J comparisons. Many of these genes also exhibited expression differences between males and females within a strain.

Conclusions: This study utilised data from both male and female animals to identify a number of genes that may be involved in the development of hypertension. We show that female data can be used to refine candidate genes and pathways, as well as highlight potential mechanisms to explain the differences in prevalence and severity of disease between men and women.

Keywords: Schlager mouse, Genome wide gene expression

\section{Background}

Essential hypertension is a leading cause of morbidity and mortality worldwide. Risk factors for the development of hypertension include a combination of environmental lifestyle factors as well as genetic factors. Significant sex differences in incidence and severity have long been recognized in the prevalence of hypertension [1]. These differences between males and females have suggested that the pathogenesis of human hypertension is modified by sex. The underlying mechanism that leads to disease may however be common to both sexes. The majority of studies utilising animal models of disease are carried out in

\footnotetext{
* Correspondence: c.chiu@uws.edu.au

'School of Medicine, University of Western Sydney, Locked Bag 1797, Penrith, NSW 2751, Australia

Full list of author information is available at the end of the article
}

male animals only, which does not allow for identification of common pathways in both sexes.

The Schlager hypertensive mouse model, (BPH/2J) and its normotensive control (BPN/3J) are a model of spontaneous hypertension that were derived via selective breeding for high and normal blood pressure. The BPH/2J strain mimics human disease with elevated blood pressure, increased heart rate, and early mortality. Previous studies have investigated gene expression changes in $\mathrm{BPH} / 2 \mathrm{~J}$ and BPN/3J male mice [2,3]. One study identified expression changes during early and established hypertension in the hypothalamus [2]. At both time points, genes involved in inflammation and oxidative stress were identified as being differentially expressed between BPH/2J and BPN/3J animals. Puig et al. compared gene expression in kidney, 
aorta, heart and liver tissue in male $\mathrm{BPH} / 2 \mathrm{~J}, \mathrm{BPN} / 3 \mathrm{~J}$ and the BPL/1 J (low blood pressure) strains. They identified 222 transcripts that were differentially expressed across all tissues with expression changes in the same direction [3]. Whether genes and pathways with altered expression in male hypertensive mice are also altered in female mice, remains to be determined.

In the current study we performed gene expression arrays in kidney tissue from male and female $\mathrm{BPH} / 2 \mathrm{~J}$ and $\mathrm{BPN} / 3 \mathrm{~J}$ mice. We compared gene expression profiles between $\mathrm{BPH} / 2 \mathrm{~J}$ and $\mathrm{BPN} / 3 \mathrm{~J}$ strains, and identified genes that were common to both sexes. Focusing on biological processes common to both males and females will help to refine the mechanisms that are involved in the development of hypertension.

\section{Methods}

\section{Blood pressure measurements}

This study involved Schlager BPH/2J and BPN/3J mice. Previous radiotelemetry studies on 18 to 20 week old males have shown that blood pressure of $\mathrm{BPH} / 2 \mathrm{~J}$ hypertensive mice is consistently high and $\mathrm{BPN} / 3 \mathrm{~J}$ mice is consistently normal [4]. In this study, radiotelemetry was performed on 12 to 13 week old male $(B P N / 3 J n=8$, $\mathrm{BPH} / 2 \mathrm{~J} \mathrm{n}=6)$ and female $(\mathrm{BPN} / 3 \mathrm{~J} \mathrm{n}=3, \mathrm{BPH} / 2 \mathrm{~J} \mathrm{n}=4$ ) mice as previously described $[4,5]$. In brief, blood pressure telemetry transmitters were implanted, and 10 days after surgery recordings of systolic arterial pressure were obtained. This had the Alfred Medical Research Education Precinct Animal Ethics Committee approval.

\section{Tissue collection}

To avoid introducing gene expression changes due to stress handling from blood pressure measurement, blood pressure was not directly measured in the animals used for the microarrays. Kidney tissue was collected from 12 week old male and female hypertensive $\mathrm{BPH} / 2 \mathrm{~J}$ and age-matched normotensive BPN/3J mice ( $n=6 /$ group) during the inactive period, which corresponds to the trough in diurnal blood pressure. Mice were euthanized via cervical dislocation and tissues were snap frozen in liquid nitrogen and stored at $-80^{\circ} \mathrm{C}$. This study had University of Western Sydney Animal Ethics Committee approval.

\section{RNA extraction}

Tissues were mechanically disrupted in the deep frozen state as previously described [6]. Total RNA was extracted from frozen tissue using the Qiagen RNeasy Mini Kit (Qiagen, Germany) according to the manufacturer's recommendations, quantified using the NanoPhotometer (Implen, Germany) and stored at $-80^{\circ} \mathrm{C}$.

\section{Microarray analysis}

RNA quality assessment and microarrays were performed by the Ramaciotti Centre for Gene Function Analysis, University of New South Wales, Australia. RNA quality was confirmed based on a RNA integrity number $>8$ using an electrophoresis bioanalyzer (2100 Agilent Bioanalyzer) and Affymetrix GeneChip Mouse Gene 1.0 ST Arrays were used. Microarray results were normalised using robust multiarray analysis (RMA). Differentially expressed genes between hypertensive and normotensive mice were identified using GenePattern 3.1 Comparative Marker Selection analysis. This analysis performs a two-sided $\mathrm{t}$-test. Genes were selected based on a Bonferroni-corrected $\mathrm{P}$ value $<0.05$. The data obtained has been deposited in the NCBI Gene Expression Omnibus (GEO) database according to the MIAME guidelines (accession number GSE54015).

\section{Gene ontology analysis}

The GENECODIS (http://genecodis.cnb.csic.es) web-based software tool was used to perform gene ontology (GO) enrichment analysis $[7,8]$. The biological process and molecular functions were analysed simultaneously. Differentially expressed genes that were common in the male and female dataset were selected and were tested against the background set of all genes present in the Affymetrix GeneChip Mouse Gene 1.0 ST Array, using the default lowest GO annotation level. In addition, pathway enrichment analysis based on the PANTHER (protein annotation through evolutionary relationship) classification database (PANTHER pathway) was used to identify significant pathways.

\section{Real-time PCR}

cDNA was synthesised using Bioscript ${ }^{\text {tm }}$ Reverse Transcriptase and random hexamers (Bioline Pty Ltd, Australia) according to the manufacturer's protocol. This was the same RNA used for the microarrays. Quantitative PCR (qPCR) was used to validate genes found to be differentially expressed on microarray. Beta Actin (bAct), glyceraldehyde 3-phosphate dehydrogenase (Gapdh), and hypoxanthine guanine phosphoribosyl transferase (Hprt1) were used as normalising genes (Additional file 1: Table S1). Individual reactions $(10 \mu \mathrm{l})$ contained $2 \times$ Sensifast SYBR Mix (Bioline), forward and reverse primers $(10 \mu \mathrm{M})$, and $4 \mu \mathrm{l}$ of cDNA (or $4 \mu \mathrm{l}$ water for no template controls). The PCR reactions were carried out in a MxPro3005P Real Time PCR System (Stratagene, Agilent Technologies, USA) followed by a dissociation curve. All samples were run in triplicate.

Cycle threshold $(\mathrm{Ct})$ values for each sample were calculated using the MxPRo QPCR software (Stratagene, Agilent Technologies, USA). Triplicate $\mathrm{Ct}$ values were averaged and the quantity $(\mathrm{Q})$ of each sample was calculated using the delta-delta $\mathrm{Ct}$ method. $\mathrm{Q}$ values from the 
normaliser genes were input into geNorm and the geometric means from these genes were used to generate a normalisation factor (NF) [9]. The Q values of the genes of interest were normalised by dividing by the NF value.

\section{Statistical analysis}

Results are expressed as mean \pm standard error, or mean fold changes in expression, and analysed using a general linear model. Statistical analysis was performed with PASW Statistics version 20. A P value of $<0.05$ was considered significant.

\section{Results}

Radiotelemetry was performed on male and female $\mathrm{BPH} / 2 \mathrm{~J}$ and BPN/3J mice at 12 to 13 weeks of age. Male $\mathrm{BPN} / 3 \mathrm{~J}$ and $\mathrm{BPH} / 2 \mathrm{~J}$ and female $\mathrm{BPH} / 2 \mathrm{~J}$ exhibited significant differences in blood pressure and heart rate, between the day (inactive period) and night (active period), with blood pressure and heart rate increased during the night (active period). No differences in day and night blood pressure or heart rate were seen in female BPN/3J. In males, systolic arterial pressure, diastolic pressure and heart rate were significantly higher in $\mathrm{BPH} / 2 \mathrm{~J}$ compared with BPN/3J mice, regardless of time of day. In females, $\mathrm{BPH} / 2 \mathrm{~J}$ mice had significantly higher systolic arterial pressure and heart rate at both day and night when compared to BPN/3J. However, no significant difference in diastolic pressure was observed between $\mathrm{BPH} / 2 \mathrm{~J}$ and BPN/3J females (Table 1). Within the BPH/2J strain no significant difference in 24 hour systolic arterial pressure or diastolic pressure or heart rate was observed between males and females. In the BPN/3J strain, no difference in 24 hour systolic arterial pressure or diastolic pressure was observed, however females had a significantly higher 24 hour heart rate $(\mathrm{p}=0.02)$ when compared to males.

Microarray expression analysis was performed on both male and female hypertensive $(\mathrm{BPH} / 2 \mathrm{~J})$ and normotensive (BPN/3J) mice. Comparison between $\mathrm{BPH} / 2 \mathrm{~J}$ and BPN/3J was performed with male and female animals analysed separately. In males, 348 genes were found to be differentially expressed between $\mathrm{BPH} / 2 \mathrm{~J}$ and $\mathrm{BPN} / 3 \mathrm{~J}$ (Additional file 2: Table S2). Of these, 170 were upregulated and 178 were downregulated in the $\mathrm{BPH} / 2 \mathrm{~J}$. In the females, 300 genes were found to be differentially expressed, with 152 upregulated and 150 downregulated in the $\mathrm{BPH} / 2 \mathrm{~J}$ (Additional file 3: Table S3). Of the genes that were identified as being differentially expressed, 70 upregulated and 83 downregulated genes were common between males and females.

Of the differentially expressed genes common in both the male and female lists, GO enrichment analysis was performed using GENECODIS [7,8] and biological processes and molecular functions were examined. Single categories that were significantly enriched in our gene
Table 1 Radiotelemetry measurements in 12 to 13 week old $\mathrm{BPH} / 2 \mathrm{~J}$ and $\mathrm{BPN} / 3 \mathrm{~J}$ mice

\begin{tabular}{llll}
\hline & $\begin{array}{l}\text { BPN/3J } \\
\text { (mean } \pm \mathbf{S E} \text { ) }\end{array}$ & $\begin{array}{l}\text { BPH/2J } \\
\text { (mean } \pm \mathbf{S E} \text { ) }\end{array}$ & p value \\
\hline Male & $\mathbf{n}=\mathbf{6}$ & $\mathbf{n = 8}$ & \\
Systolic BP - Day (mmHg) & $105.7 \pm 1.8$ & $133.5 \pm 2.9$ & $<0.001$ \\
Systolic BP- Night (mmHg) & $114.2 \pm 1.4$ & $154.4 \pm 4.2$ & $<0.001$ \\
Systolic BP - 24 hr (mmHg) & $109.9 \pm 0.8$ & $143.9 \pm 4.0$ & $<0.001$ \\
Diastolic BP - Day (mmHg) & $78.2 \pm 2.0$ & $100.3 \pm 3.2$ & $<0.001$ \\
Diastolic BP - Night (mmHg) & $87.0 \pm 0.7$ & $116.5 \pm 3.3$ & $<0.001$ \\
Diastolic BP - 24 hr (mmHg) & $82.6 \pm 0.6$ & $108.4 \pm 1.3$ & $<0.001$ \\
Heart rate - Day (beats/min) & $358.7 \pm 13.8$ & $518.1 \pm 26.3$ & 0.001 \\
Heart rate - Night (beats/min) & $438.5 \pm 16.3$ & $627.3 \pm 5.0$ & $<0.001$ \\
Heart rate - 24 hr (beats/min) & $398.6 \pm 5.2$ & $572.7 \pm 25.2$ & $<0.001$ \\
Female & $\mathbf{n}=\mathbf{3}$ & $\mathbf{n}=\mathbf{4}$ & \\
Systolic BP - Day (mmHg) & $112.1 \pm 1.4$ & $128.5 \pm 1.5$ & 0.008 \\
Systolic BP- Night (mmHg) & $117.6 \pm 3.8$ & $148.3 \pm 1.6$ & 0.009 \\
Systolic BP - 24 hr (mmHg) & $115.4 \pm 3.6$ & $138.4 \pm 1.2$ & 0.009 \\
Diastolic BP - Day (mmHg) & $93.4 \pm 2.3$ & $93.5 \pm 2.1$ & 0.98 \\
Diastolic BP - Night (mmHg) & $97.3 \pm 6.6$ & $111.7 \pm 1.8$ & 0.17 \\
Diastolic BP - 24 hr (mmHg) & $95.3 \pm 3.6$ & $102.6 \pm 0.8$ & 0.36 \\
Heart rate - Day (beats/min) & $486.8 \pm 15.5$ & $551.7 \pm 9.7$ & 0.018 \\
Heart rate - Night (beats/min) & $505.8 \pm 21.1$ & $677.7 \pm 7.4$ & 0.001 \\
Heart rate - 24 hr (beats/min) & $496.3 \pm 11.2$ & $614.7 \pm 7.3$ & 0.022 \\
\hline & & &
\end{tabular}

set were transport $(\mathrm{GO}: 0006810, \mathrm{p}=0.01)$, metabolic process $(\mathrm{GO}: 0008152, \mathrm{p}=0.019)$ and hydrolase activity (GO:0016787, $p=0.038$ ). In addition to these single GO terms, a significant set of genes were co-annotated with oxidation-reduction process and oxidoreductase activity (GO:0055114, GO:0016491, p = 0.026) or metabolic process and hydrolase activity (GO:0008152, GO:0016787, $\mathrm{p}=0.025)$. Panther Pathway enrichment analysis was also performed and no pathways were found to be significantly represented in our gene list.

Genes were selected for validation by qPCR (Table 2). These genes were selected based on fold change differences, previous association with blood pressure regulation, and/or involvement in processes or pathways that may influence blood pressure. Of the genes that were differentially expressed in both the male and female, $\mathrm{BPH} / 2 \mathrm{~J}$ and BPN/3J comparisons, 15 out of 21 genes (71\%) were validated on qPCR. The expression of these genes was also compared between males and females within a strain (Table 3). The expression of $H d c, E d n 3$, IllOrb, Gabra3a, Kcnk1, and Serpinb8 was significantly higher in female kidneys compared to male kidneys in both the BPH/2J and BPN/3J strains. Angplt7, Cndp2 and Cyp $4 b 1$ expression was significantly lower in females when compared to males within a strain. The expression of Pld1 and Pak6 was significantly higher only in $\mathrm{BPH} / 2 \mathrm{~J}$ 
Table 2 Genes common in males and females that were validated by qPCR

\begin{tabular}{|c|c|c|c|c|c|c|}
\hline \multirow[b]{2}{*}{ Gene } & \multicolumn{3}{|l|}{ Male } & \multicolumn{3}{|c|}{ Female } \\
\hline & $\begin{array}{l}\mathrm{FC} \\
\mathrm{qPCR}\end{array}$ & $\begin{array}{l}P \text { value } \\
\text { qPCR }\end{array}$ & $\begin{array}{l}\text { FC } \\
\text { (array) }\end{array}$ & $\begin{array}{l}\text { FC } \\
\text { qPCR }\end{array}$ & $\begin{array}{l}\text { P value } \\
\text { qPCR }\end{array}$ & $\begin{array}{l}\text { FC } \\
\text { (array) }\end{array}$ \\
\hline $\mathrm{Hdc}$ & 1154 & $<0.001$ & 1.86 & 5.0 & $<0.001$ & 1.10 \\
\hline Gabra3 & 97 & $<0.001$ & 1.53 & 128 & $<0.001$ & 1.56 \\
\hline Angptl7 & 53 & $<0.001$ & 1.47 & 89 & $<0.001$ & 1.68 \\
\hline Cyp4b1 & 12.2 & $<0.001$ & 1.15 & 20.4 & $<0.001$ & 1.39 \\
\hline Edn3 & 4.4 & $<0.001$ & 1.14 & 3.8 & $<0.001$ & 1.16 \\
\hline Dna2 & 1.7 & 0.001 & 1.12 & 1.4 & 0.02 & 1.08 \\
\hline Cndp2 & 1.4 & 0.01 & 1.12 & 1.9 & 0.03 & 1.08 \\
\hline Mcm6 & -10 & $<0.001$ & -1.30 & -8.7 & $<0.001$ & -1.30 \\
\hline Vwal & -10 & $<0.001$ & -1.23 & -3.0 & $<0.001$ & -1.14 \\
\hline Kcnk1 & -3.3 & $<0.001$ & -1.15 & -3.2 & $<0.001$ & -1.18 \\
\hline Serpinb8 & -2.5 & $<0.001$ & -1.21 & -2.7 & $<0.001$ & -1.22 \\
\hline Pld 1 & -2.0 & 0.001 & -1.11 & -1.4 & 0.02 & -1.11 \\
\hline Pter & -1.7 & 0.001 & -1.03 & -1.5 & $<0.001$ & -1.03 \\
\hline Pak6 & -1.7 & $<0.001$ & -1.10 & -1.3 & 0.03 & -1.11 \\
\hline /l10rb & -1.3 & 0.004 & -1.07 & -1.3 & 0.01 & -1.06 \\
\hline
\end{tabular}

Values represent mean fold changes (FC) between $\mathrm{BPH} / 2 \mathrm{~J}$ and $\mathrm{BPN} / 3 \mathrm{~J}$ samples relative to $\mathrm{BPN} / 3 \mathrm{~J}$. Positive values indicate higher expression in $\mathrm{BPH} / 2 \mathrm{~J}$ samples while, negative values indicate lower expression in $\mathrm{BPH} / 2 \mathrm{~J}$ samples.

Table 3 Quantitative PCR fold changes in females, relative to males within a strain

\begin{tabular}{|c|c|c|c|c|}
\hline \multirow[b]{2}{*}{ Gene } & \multicolumn{2}{|c|}{$\mathrm{BPH} / 2 \mathrm{~J}$} & \multicolumn{2}{|c|}{ BPN/3J } \\
\hline & $\overline{F C}$ & $P$ value & $\overline{F C}$ & $P$ value \\
\hline $\mathrm{Hdc}$ & 1.7 & $<0.001$ & 388 & $<0.001$ \\
\hline Gabra3 & 1.6 & 0.005 & 1.2 & 0.01 \\
\hline Angptl7 & -2.8 & $<0.001$ & -4.7 & $<0.001$ \\
\hline Cyp4b1 & -13.2 & $<0.001$ & -22.2 & $<0.001$ \\
\hline Edn3 & 1.7 & 0.005 & 1.9 & 0.005 \\
\hline Dna2 & 1.0 & 0.17 & 1.2 & 0.99 \\
\hline Cndp2 & -1.6 & $<0.001$ & -2.1 & 0.002 \\
\hline Mcm6 & 1.9 & 0.03 & 1.7 & 0.03 \\
\hline Vwal & 1.8 & 0.01 & -1.5 & $<0.001$ \\
\hline Kcnk1 & 1.9 & $<0.001$ & 2.1 & $<0.001$ \\
\hline Serpinb8 & 1.8 & $<0.001$ & 1.8 & 0.001 \\
\hline Pld 1 & 1.9 & 0.001 & 1.4 & 0.03 \\
\hline Pter & 1.2 & 0.12 & 1.2 & 0.08 \\
\hline Pak6 & 1.3 & 0.01 & 1.1 & 0.38 \\
\hline Il10rb & 1.6 & $<0.001$ & 1.7 & $<0.001$ \\
\hline
\end{tabular}

Values represent mean fold changes (FC) between male and female samples relative to males within a strain. Positive values indicate higher expression in female samples while, negative values indicate lower expression in female samples. females, while the expression of $V w a 1$ was significantly lower only in the BPN/3J females. No difference in expression between the sexes within a strain was observed for Mcm6, Dna2 or Pter.

\section{Discussion}

This study performed gene expression arrays in kidneys of $\mathrm{BPH} / 2 \mathrm{~J}$ and $\mathrm{BPN} / 3 \mathrm{~J}$ male and female mice. It is the first study to measure and compare gene expression profiles in female $\mathrm{BPH} / 2 \mathrm{~J}$ and $\mathrm{BPN} / 3 \mathrm{~J}$ mice to determine the mechanisms involved in the development of high blood pressure in these mice. The $\mathrm{BPH} / 2 \mathrm{~J}$ strain was generated via multiple generations of brother sister mating selecting for high blood pressure. Radiotelemetry in these mice found significantly increased systolic arterial pressure in both male and female hypertensive mice, compared with the normotensive strain, with no difference in blood pressure between males and females. This would suggest that a common mechanism is responsible for high blood pressure in both sexes. We found that while there were differences in the expression profiles between $\mathrm{BPH} / 2 \mathrm{~J}$ and BPN/3J depending on sex, approximately half of the differentially expressed genes were present in both male and female datasets. This allowed us to refine the list of possible candidate genes that were only differentially expressed in both sexes. This list included Angptl7, Hdc, Cndp2, Dna2, $E d n 3$, which were up-regulated in the hypertensive mice and may have a physiological role in the regulation of high blood pressure. Mcm6 and Il1Orb were both downregulated in the hypertensive mice and may also play a role high blood pressure. A previous study compared gene expression across multiple tissues between 12 week old male hypertensive and normotensive mice [3]. A number of genes, including Angplt7, Hdc, Mcm6 and Il1Orb that were identified as being differentially expressed in the kidneys of those animals were also identified in the kidneys of male and females in the current study.

We show that expression of this Angptl7 is increased in $\mathrm{BPH} / 2 \mathrm{~J}$ kidneys, with expression being significantly higher in male kidneys compared to females within a strain. Angptl7 belongs to the angiopoietin like protein family. Several angiopoietin-like proteins have been shown to be involved in the regulation of angiogenesis as well as lipid and glucose metabolism [10]. Angptl7 is the least studied of all the angiopoietin-like proteins. Studies in patients with glaucoma, a condition characterised by increased intraocular pressure, have reported elevated Angptl7 expression in the cornea [11]. Longitudinal cohort studies have also shown hypertension is associated with an elevated risk of developing glaucoma [12]. These studies, together with the increased expression of Angptl7 observed in the kidneys of our hypertensive mice, suggests that Angptl7 may be upregulated in response to increased vasculature pressure. 
The BPH/2J has been shown to be a model of neurogenic hypertension and these mice have increased sympathetic activity [4]. Histidine decarboxylase (Hdc) and carnosine dipeptidase 2 (Cndp2) have been shown to be involved in sympathetic activity. These genes are part of the carosine- histidine - histamine metabolic pathway. Carnosine is broken down into histidine via the action of Cndp2, and then histidine is converted into histamine by Hdc. Carnosine is an antioxidant that has been reported to have a protective effect on kidneys as a scavenger of reactive oxygen species [13]. It has been shown to reduce renal sympathetic nerve activity in rats, and inhibit hypertension in DOCA salt hypertensive rats [14]. Additionally, a patient with congenital carnosinase deficiency, the inability to breakdown carnosine, was reported to have elevated levels of carnosine and hypotension [15]. Cndp2 expression was significantly elevated in both $\mathrm{BPH} / 2 \mathrm{~J}$ males and females. Elevated levels of $C n d p 2$ may result in reduced carnosine, decreased protection against oxidative stress, and increased levels of histidine.

Hdc catalyses the conversion of histidine to histamine. Histamine is a neurotransmitter that is involved in the regulation of renal sympathetic activity and response to inflammation. In anesthetised rats a low dose of histamine suppressed renal sympathetic nerve activity and blood pressure, while a high dose of histamine elevated renal sympathetic nerve activity and blood pressure [16]. $H d c$ was previously reported to be significantly upregulated in the kidneys of 12 week male $\mathrm{BPH} / 2 \mathrm{~J}$ [3]. It is upregulated by estrogen and is expressed at higher levels in female kidneys compared to males [17]. This was reflected in our study, where the fold change between male $\mathrm{BPH} / 2 \mathrm{~J}$ and $\mathrm{BPN} / 3 \mathrm{~J}$ was significantly larger than the fold change between female $\mathrm{BPH} / 2 \mathrm{~J}$ and $\mathrm{BPN} / 3 \mathrm{~J}$, due to the BPN/3J males having low $H d c$ expression. Increased expression of $C n d p 2$ and $H d c$ may result in higher levels of histamine in the $\mathrm{BPH} / 2 \mathrm{~J}$ animals which may contribute to the elevated sympathetic activity and blood pressure seen in this strain.

Genes that were involved in DNA replication, repair and telomere maintenance were identified in both male and female datasets. Mcm6 is a subunit of the Mcm2-7 complex, a replicative helicase that is involved in eukaryotic DNA replication. It is an essential component of the MCM complex as mutations in Mcm6 result in the loss of helicase activity [18]. DNA2 is a nuclease/helicase that is essential for Okazaki fragment processing during DNA replication. Recent studies have also suggested a role for Mcm6 and Dna2 in telomere length maintenance [19-21]. As telomere length has been associated with hypertension [22], dysregulation in the expression of these genes may affect DNA replication and telomere maintenance may contribute the development of disease.
A number of the differentially expressed genes in both males and females were known to have links to blood pressure regulation. $E d n 3$ was identified as a candidate gene for hypertension in humans via a GWA study [23]. The expression of $E d n 3$ was found to be upregulated in $\mathrm{BPH} / 2 \mathrm{~J}$ animals. Females were also found to have significantly higher $E d n 3$ expression when compared to males within a strain. Increased expression of $E d n 3$ in the kidneys of ageing rats has suggested that it may be a marker for advanced ageing [24]. The prevalence of hypertension increases with increasing age [25]. This may suggest a role for premature ageing in hypertension.

Inflammation and oxidative stress are characteristic of hypertension. IL10 is an anti-inflammatory cytokine that can inhibit the synthesis of pro-inflammatory cytokines [26]. It functions by binding to its receptor IL10R and activates JAK and STAT pathways. DNA variants in IL1Orb, a subunit of IL10R, have been associated with ischemic stroke in individuals with hypertension [27], and IL10 deficiency has been associated with preeclampsia, a condition characterised by high blood pressure during pregnancy [28,29]. The reduced expression of $I L 10 \mathrm{rb}$ seen in both male and female BPH/2J mice may result in reduced IL10 activity and increased inflammation in the hypertensive animals.

Many of the genes that were differentially expressed between $\mathrm{BPH} / 2 \mathrm{~J}$ and $\mathrm{BPN} / 3 \mathrm{~J}$ also exhibited expression differences between males and females within a strain. These differences suggests that while a common mechanism is most likely responsible for high blood pressure in the $\mathrm{BPH} / 2 \mathrm{~J}$ strain, differences in sex hormones will influence how gene/pathways respond to elevated blood pressure. Furthermore, these differences in expression may help to explain the differences in disease phenotype that are seen between men and women.

\section{Conclusion}

This study has identified a combination of potential new candidate genes with no currently known links to hypertension, as well as genes that had been previously associated with hypertension and blood pressure control. The genes that we identified in both male and female BPH/2J hypertensive mice may be involved in the development or maintenance of hypertension. Our findings from the female dataset have helped to refine the possible pathways that are involved in the onset of hypertension in these animals, as well as highlight potential mechanisms that may explain the differences that are seen in the prevalence and severity of hypertension between men and women.

\section{Grants}

CLC received a University of Western Sydney Early Career Seed Grant to undertake this project. JM Lind is supported by a National Health and Medical Research - Australian Biomedical Fellowship. 


\section{Additional files}

Additional file 1: Table S1. Primer sequences.

Additional file 2: Table S2. Differentially expressed genes in the kidneys of 12 week old $\mathrm{BPH} / 2 \mathrm{~J}$ males, relative to BPN/3J males, using Bonferroni corrected $p<0.05$

Additional file 3: Table S3. Differentially expressed genes in the kidneys of 12 week old $\mathrm{BPH} / 2 \mathrm{~J}$ females, relative to BPN/3J females, using Bonferroni corrected $p<0.05$

\section{Competing interests}

The authors declare that they have no competing interest.

\section{Authors' contributions}

CLC participated in study design, laboratory work, data analysis and interpretation, and manuscript writing. KLJ participated in data analysis and interpretation. NLH and NS participated in laboratory work. GAH participated in data analysis and interpretation, manuscript editing. JML participated in study design, data interpretation and manuscript writing. All authors read and approved the final manuscript.

\section{Acknowledgements}

The authors would like to thank the UWS School of Medicine Animal Facility staff for their assistance in animal care and tissue collection.

\section{Author details}

${ }^{1}$ School of Medicine, University of Western Sydney, Locked Bag 1797, Penrith, NSW 2751, Australia. ${ }^{2}$ Neuropharmacology Laboratory, Baker IDI Heart and Diabetes Research Institute, Victoria, Australia. ${ }^{3}$ Department of Pharmacology, Monash University, Victoria, Australia.

Received: 19 February 2014 Accepted: 19 August 2014 Published: 30 August 2014

\section{References}

1. Chen YF: Sexual dimorphism of hypertension. Curr Opin Nephrol Hypertens 1996, 5(2):181-185.

2. Marques FZ, Campain AE, Davern PJ, Yang YH, Head GA, Morris BJ: Global identification of the genes and pathways differentially expressed in hypothalamus in early and established neurogenic hypertension. Physiol Genomics 2011, 43(12):766-771.

3. Puig $O$, Wang IM, Cheng P, Zhou P, Roy S, Cully D, Peters M, Benita $Y$, Thompson J, Cai TQ: Transcriptome profiling and network analysis of genetically hypertensive mice identifies potential pharmacological targets of hypertension. Physiol Genomics 2010, 42A(1):24-32.

4. Davern PJ, Nguyen-Huu TP, La Greca L, Abdelkader A, Head GA: Role of the sympathetic nervous system in Schlager genetically hypertensive mice. Hypertension 2009, 54(4):852-859.

5. Jackson KL, Marques FZ, Watson AM, Palma-Rigo K, Nguyen-Huu TP, Morris BJ, Charchar FJ, Davern PJ, Head GA: A novel interaction between sympathetic overactivity and aberrant regulation of renin by miR-181a in $\mathrm{BPH} / 2 \mathrm{~J}$ genetically hypertensive mice. Hypertension 2013, 62(4):775-781.

6. Chiu CL, Morgan CT, Lupton SJ, Lind JM: Parent of origin influences the cardiac expression of vascular endothelial growth factor (Vegfa). BMC Med Genet 2013, 14:43.

7. Tabas-Madrid D, Nogales-Cadenas R, Pascual-Montano A: GeneCodis3: a non-redundant and modular enrichment analysis tool for functional genomics. Nucleic Acids Res 2012, 40(Web Server issue):W478-W483.

8. Carmona-Saez P, Chagoyen M, Tirado F, Carazo JM, Pascual-Montano A: GENECODIS: a web-based tool for finding significant concurrent annotations in gene lists. Genome Biol 2007, 8(1):R3.

9. Vandesompele J, De Preter K, Pattyn F, Poppe B, Van Roy N, De Paepe A Speleman F: Accurate normalization of real-time quantitative RT-PCR data by geometric averaging of multiple internal control genes. Genome Biol 2002, 3(7):RESEARCH0034.

10. Katoh $Y$, Katoh M: Comparative integromics on Angiopoietin family members. Int J Mol Med 2006, 17(6):1145-1149.
11. Comes N, Buie LK, Borras T: Evidence for a role of angiopoietin-like 7 (ANGPTL7) in extracellular matrix formation of the human trabecular meshwork: implications for glaucoma. Genes Cells 2011, 16(2):243-259.

12. Newman-Casey PA, Talwar N, Nan B, Musch DC, Stein JD: The relationship between components of metabolic syndrome and open-angle glaucoma. Ophthalmology 2011, 118(7):1318-1326.

13. Riedl E, Pfister F, Braunagel $M$, Brinkkotter $P$, Sternik $P$, Deinzer $M$, Bakker SJ, Henning RH, van den Born J, Kramer BK, Navis G, Hammes HP, Yard B, Koeppel $\mathrm{H}$ : Carnosine prevents apoptosis of glomerular cells and podocyte loss in STZ diabetic rats. Cell Physiol Biochem 2011, 28(2):279-288.

14. Niijima A, Okui T, Matsumura Y, Yamano T, Tsuruoka N, Kiso Y, Nagai K Effects of L-carnosine on renal sympathetic nerve activity and DOCA-salt hypertension in rats. Auton Neurosci 2002, 97(2):99-102.

15. Willi SM, Zhang Y, Hill JB, Phelan MC, Michaelis RC, Holden KR: A deletion in the long arm of chromosome 18 in a child with serum carnosinase deficiency. Pediatr Res 1997, 41(2):210-213.

16. Tanida M, Kaneko $\mathrm{H}$, Shen J, Nagai K: Involvement of the histaminergic system in renal sympathetic and cardiovascular responses to leptin and ghrelin. Neurosci Lett 2007, 413(1):88-92.

17. Morgan TK, Montgomery K, Mason V, West RB, Wang L, van de Rijn M, Higgins JP: Upregulation of histidine decarboxylase expression in superficial cortical nephrons during pregnancy in mice and women. Kidney Int 2006, 70(2):306-314.

18. You Z, Komamura Y, Ishimi Y: Biochemical analysis of the intrinsic Mcm4-Mcm6-Mcm7 DNA helicase activity. Mol Cell Biol 1999, 19(12):8003-8015.

19. Chai $W$, Zheng $L$, Shen $B$ : DNA2, a new player in telomere maintenance and tumor suppression. Cell Cycle 2013, 12(13):1985-1986.

20. Lin W, Sampathi S, Dai H, Liu C, Zhou M, Hu J, Huang Q, Campbell J, Shin-Ya K, Zheng L, Chai W, Shen B: Mammalian DNA2 helicase/nuclease cleaves G-quadruplex DNA and is required for telomere integrity. EMBO J 2013, 32(10):1425-1439.

21. Ungar L, Yosef N, Sela Y, Sharan R, Ruppin E, Kupiec M: A genome-wide screen for essential yeast genes that affect telomere length maintenance. Nucleic Acids Res 2009, 37(12):3840-3849.

22. Fuster JJ, Diez J, Andres V: Telomere dysfunction in hypertension. $J$ Hypertens 2007, 25(11):2185-2192

23. Levy D, Ehret GB, Rice K, Verwoert GC, Launer LJ, Dehghan A, Glazer NL, Morrison AC, Johnson AD, Aspelund T, Aulchenko Y, Lumley T, Köttgen A, Vasan RS, Rivadeneira F, Eiriksdottir G, Guo X, Arking DE, Mitchell GF, Mattace-Raso FU, Smith AV, Taylor K, Scharpf RB, Hwang SJ, Sijbrands EJ, Bis J, Harris TB, Ganesh SK, O'Donnell CJ, Hofman A: Genome-wide association study of blood pressure and hypertension. Nat Genet 2009, 41(6):677-687.

24. Lattmann T, Shaw S, Munter K, Vetter W, Barton M: Anatomically distinct activation of endothelin-3 and the L-arginine/nitric oxide pathway in the kidney with advanced aging. Biochem Biophys Res Commun 2005, 327(1):234-241.

25. Ostchega Y, Dillon CF, Hughes JP, Carroll M, Yoon S: Trends in hypertension prevalence, awareness, treatment, and control in older U.S. adults: data from the National Health and Nutrition Examination Survey 1988 to 2004. J Am Geriatr Soc 2007, 55(7):1056-1065.

26. Fiorentino DF, Zlotnik A, Vieira P, Mosmann TR, Howard M, Moore KW, O'Garra A: IL-10 acts on the antigen-presenting cell to inhibit cytokine production by Th1 cells. J Immunol 1991, 146(10):3444-3451.

27. Park HK, Kim DH, Yun DH, Ban JY: Association between IL10, IL10RA, and IL10RB SNPs and ischemic stroke with hypertension in Korean population. Mol Biol Rep 2013, 40(2):1785-1790.

28. Kalkunte $S$, Nevers T, Norris WE, Sharma S: Vascular IL-10: a protective role in preeclampsia. J Reprod Immunol 2011, 88(2):165-169.

29. Orange $S$, Horvath J, Hennessy A: Preeclampsia is associated with a reduced interleukin-10 production from peripheral blood mononuclear cells. Hypertens Pregnancy 2003, 22(1):1-8.

doi:10.1186/s12881-014-0101-x

Cite this article as: Chiu et al:: Identification of genes with altered expression in male and female Schlager hypertensive mice. BMC Medical Genetics 2014 15:101. 